\title{
CARACTERISTICAS EPIDEMIOLOGICAS DE LA MORTALIDAD NEONATAL INSTITUTO NACIONAL MATERNO PERINATAL 2015 - 2016
}

\author{
Carmen Dávila Aliaga¹, Elina Mendoza Ibañez ${ }^{2}$
}

\begin{abstract}
RESUMEN
Objetivo: Determinar las causas de mortalidad neonatal y sus características epidemiológicas en nuestra Institución, 2015 y 2016. Materiales y métodos: Estudio retrospectivo, descriptivo y observacional, se revisaron las historias clínicas de pacientes fallecidos en el departamento de neonatología del INMP durante 2015 y 2016. Resultados: El 2015 tuvimos 21729 recién nacidos vivos (rnv) y 234 fallecidos. El 2016 nacieron 22060 y fallecieron 290. Para ambos períodos la edad materna fue principalmente de 18 a 35 años, los lugares de procedencia más frecuente fue Cercado y San Juan de Lurigancho, la vía de nacimiento más frecuente fue cesárea. El 2015, el 56\% fue varón y en el 2016 no hubo diferencias de género. Los menores de 37 semanas de edad gestacional (EG) en el 2015 fueron $71 \%$ y el 2016 73\%, predominando los menores de 28 semanas y de 34 - 36 semanas de EG. El 2015, 68,4\% era menor de 7 días de vida en relación al $67,9 \%$ el 2016; destacando los fallecidos dentro de las primeras 24 horas de vida. Los menores de $1500 \mathrm{gr}$ al nacer fueron $45 \%$ (2015) y 50,7\% (2016). La causa básica de fallecimiento en ambos años fueron malformaciones congénitas, infecciones, asfixia y/o evento hipóxico severo y prematuridad en el orden señalado. Las principales malformaciones encontradas son las del sistema nervioso, aparato digestivo y cardiovascular. Conclusiones. La cifra de fallecidos se incrementó en 19,3\% entre 2015 al 2016, aumento relacionado a malformaciones congénitas y prematuridad extrema, identificándose como causas reducibles de fallecimiento las infecciones y asfixia neonatal.
\end{abstract}

Palabras clave: Mortalidad neonatal; Mortalidad postneonatal; Mortalidad neonatal tardía (Fuente DeCS BIREME).

\section{EPIDEMIOLOGICAL CHARACTERISTICS OF NEONATAL MORTALITY IN THE NATIONAL PERINATAL MATERNAL INSTITUTE INMP 2015 - 2016}

\begin{abstract}
Objective: To determine the causes of neonatal mortality and its epidemiological characteristics in our institution, 2015 and 2016. Materials and methods: Retrospective, descriptive and observational study, we reviewed the clinical records of patients who died in the neonatal department of the INMP during 2015 and 2016. Results: In 2015 we had 21729 newborns and 234 deaths. In 201622060 newborns and 290 died. For both periods the maternal age was mainly from 18 to 35 years, the places of more frequent origin were Cercado and San Juan de Lurigancho, the most frequent birth route was caesarean. In 2015, 56\% were male and in 2016 there were no gender differences. Children younger than 37 weeks of gestational age (GA) in 2015 were $71 \%$ and $201673 \%$, predominantly younger than 28 weeks and $34-36$ weeks of GA. In $2015,68,4 \%$ were less than 7 days old compared to $67,9 \%$ in 2016; mighlighting those who died within the first 24 hours of life. Children under $1500 \mathrm{gr}$ at birth were $45 \%$ (2015) and $50,7 \%$ (2016). The basic cause of death in both years were congenital malformations, infections, asphyxia and/or severe hypoxic event and prematurity in the order indicated. The main malformations found are those of the nervous system, digestive system and cardiovascular system. Conclusions: The number of deaths increased by $19,3 \%$ between 2015 and 2016 , an increase related to congenital malformations and extreme prematurity, with the identification of infections and neonatal asphyxia as reducible causes.
\end{abstract}

Key words: Neonatal mortality; Postneonatal mortality; Late neonatal mortality (Source: MeSH NLM).

\section{INTRODUCCIÓN}

La tasa de mortalidad neonatal en el Perú, ha disminuido un $67 \%$, desde 1990 hasta el 2012. (33 x 1000 nv a 21 x $1000 \mathrm{nv}^{1,2}$. OPS / OMS para el mismo período reportan una reducción del $70 \%$ (De $26,4 \times 1000 \mathrm{nv}$ a $8,3 \times 1000$ $\mathrm{nv}){ }^{3}$ Las tasas de mortalidad en países latinoamericanos como Chile, Argentina, Colombia, Bolivia, Ecuador, Venezuela oscilan entre 6 a 15 × 1000 nv.

La fracción que le corresponde a la mortalidad neonatal dentro de la mortalidad infantil, continúa siendo alta entre $40-50 \%$ en nuestro país, al igual que en otros países del continente 4 .
La mortalidad neonatal constituye un indicador que evidencia el nivel de desarrollo que tiene la atención prenatal (inadecuado control prenatal da como resultado prematuridad y bajo peso) y del recién nacido en una región.

Identificar las características de esta mortalidad, permite conocer las causales de muerte de los recién nacidos y va a permitirnos como Institución y luego como país, poder estructurar el nivel de intervención para modificar este indicador de salud pública (Mortalidad neonatal es un evento, cuya vigilancia en nuestro país está normada: N.T.S. N078-MINSA/DGE).

Médico Pediatra Neonatóloga, docente de la Universidad Nacional Federico Villareal, Directora Ejecutiva de Investigación, Docencia y Atención en Neonatología del Instituto Nacional Materno Perinatal. Lima-Perú.

Médico Pediatra Neonatóloga, Docente de la Universidad Privada San Juan Bautista, jefa del Servicio de la Unidad de Cuidados Intensivos Neonatales del Instituto Nacional Materno Perinatal. Lima-Perú

Recibido: 01-12-16 Aprobado: 30-12-16 
La experiencia demuestra que la modificación de este indicador es posible, como se evidenció, en el avance de la Neonatología de los últimos 40 años, donde intervenciones como: avance en las técnicas de ventilación mecánica neonatal, disponibilidad de surfactante pulmonar, nutrición parenteral, reanimación neonatal, manipulación mínima del recién nacido prematuro, uso regulado /medido del oxígeno, ayudó a recuperar recién nacidos prematuros que antes no sobrevivían.

Según la herramienta BABIES 15, que relaciona la variable peso al nacer y momento de defunción, se observa que el $37 \%$ de la notificación de defunciones fetales y neonatales están relacionadas a deficiencias en la salud materna; el $27 \%$ a problemas en el cuidado del embarazo, el $6 \%$ a problemas del último trimestre de embarazo y atención del parto; y un $30 \%$ está relacionada al proceso de atención y cuidados del RN dentro de la institución y en el hogar; este último porcentaje es el que tiene más posibilidades de intervención en la reducción de la mortalidad neonatal. ${ }^{2}$ La muerte del recién nacido se asocia a causas de origen multifactorial que intervienen tempranamente en la madre y su producto; factores relacionados con los genes y el medio ambiente, restricción del crecimiento intrauterino, mujeres con desnutrición crónica o anemia, embarazos múltiples e hipertensión arterial.

El objetivo del presente estudio es identificar las causas de mortalidad neonatal tanto básicas como asociadas y sus características epidemiológicas en nuestra Institución, en los años 2015 y 2016 para poder planificar las intervenciones necesarias en los niveles correspondientes, articulados con las actividades de los servicios relacionados, reducir las muertes neonatales calificadas como reducibles ò prevenibles y mejorar la calidad de atención del recién nacido. Así mismo optimizar el recojo de la información con los registros adecuados.

\section{MATERIALES Y MÉTODOS}

Estudio retrospectivo, descriptivo y observacional, en el que se revisaron todas las historias clínicas de pacientes fallecidos en el departamento de neonatología del INMP en el 2015 y 2016. El criterio de inclusión fue: Todo paciente fallecido en el departamento durante el período de estudio y el de exclusión: Todos los niños fallecidos fuera del período de estudio.

Las variables de resultado fueron la mortalidad, morbilidades neonatales al momento del fallecimiento y la causa básica de fallecimiento. Las morbilidades asociadas fueron síndrome de dificultad respiratoria (enfermedad de membrana hialina), neumonía, hemorragia pulmonar, insuficiencia respiratoria, neumotórax, displasia broncopulmonar (DBP: oxígeno más de 28 días), ductus arterioso permeable (PCA), sepsis, shock séptico, enterocolitis necrotizante (NEC), hemorragia intracraneana, hemorragia intraventricular, leucomalacia periventricular (LPV), trastorno de coagulación, síndrome convulsivo, insuficiencia renal, anemia, y disglicemia. Malformaciones congénitas mayores (gastroquisis, onfalocele, malformación adenomatoidea quística, atresia intestinal, cardiopatía congénita, hidrocefalia, hidranancefalia, acranea, etc). Las causas básicas de fallecimiento se agruparon en: malformaciones congénitas mayores, infecciones, asfixia y/o evento hipóxico perinatal severo, prematuridad e insuficiencia respiratoria. Las variables independientes fueron edad materna, distrito de procedencia, terminación del embarazo (vaginal ó cesárea), edad gestacional, sexo (masculino/femenino), peso de nacimiento (gramos), edad al momento de fallecimiento. La información obtenida fue consignada en una ficha para la investigación.

\section{RESULTADOS}

En el año 2015 tuvimos 21729 recién nacidos vivos y 234 fallecidos en el departamento de neonatología, 203 fallecidos de 28 días o menos, sí sólo consideramos los mayores de 22 semanas de EG y peso mayor de 500 gr tenemos 186 fallecidos, lo cual corresponden a una tasa de mortalidad neonatal de 8,56 x 1000 rnv; además hubieron 31 fallecidos mayores de 28 días. En el año 2016 tuvimos 22060 recién nacidos vivos y 290 fallecidos; 251 fallecieron a los 28 días o menos, sí retiramos los menores de 22 semanas y menores de 500 g, quedan 244 rnv, obteniendo una tasa de mortalidad neonatal de 11.06 x 1000 rnv; además hubieron 39 fallecidos mayores de 28 días. Gráficos 1, 2 y 3.

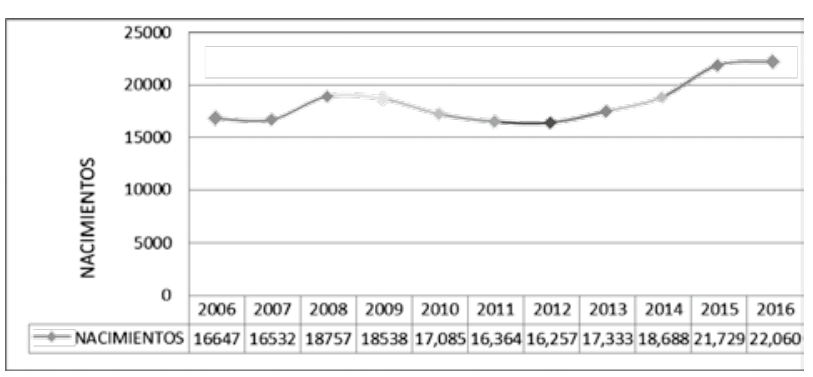

Gráfico 1. Total de nacimientos INMP 2006 -2016

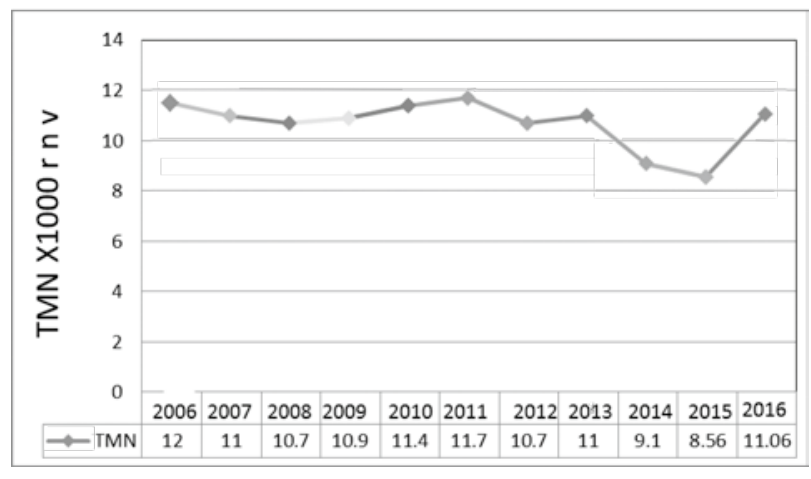

Gráfico 2. Tasa de mortalidad neonatal INMP 2007 -2016 


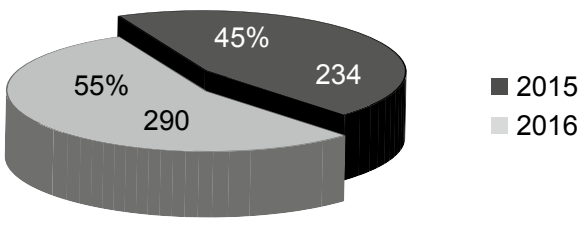

Grafico 3. Fallecidos en el departamento de neonatología - INMP 2007 -2016

Los resultados reportados corresponden al análisis del $100 \%$ de fallecidos durante el período de estudio.

Tabla 1. Edad materna de niños fallecidos INMP 2015 $-2016$

\begin{tabular}{cccccccc}
\hline \multirow{2}{*}{ Años } & \multicolumn{2}{c}{$\begin{array}{c}<18 \\
\text { años }\end{array}$} & \multicolumn{2}{c}{$\begin{array}{c}\mathbf{1 8}-\mathbf{3 5} \\
\text { años }\end{array}$} & \multicolumn{2}{c}{$>$ 35 años } & $\begin{array}{c}\text { Total } \\
\text { fallecidos }\end{array}$ \\
\cline { 2 - 8 } & $\mathbf{N}^{\circ}$ & $\%$ & $\mathrm{~N}^{\circ}$ & $\%$ & $\mathrm{~N}^{\circ}$ & $\%$ & $\mathrm{~N}^{\circ}$ \\
\hline 2015 & 18 & 8 & 170 & $\mathbf{7 3}$ & 46 & 19 & 234 \\
\hline 2016 & 9 & 3 & 237 & $\mathbf{8 2}$ & 44 & 15 & 290 \\
\hline
\end{tabular}

En la Tabla 1 estratificamos la edad materna de los recién nacidos fallecidos como menores de 18 años, de 18 a 35 años y mayores de 35 años. El $73 \%$ en el 2015 y $82 \%$ en el 2016 fueron hijos de madres entre 18 a 35 años.

La distribución por procedencia mostro para ambos años ser a predominio del cercado de Lima 23\% para el 2015 y $18 \%$ para el 2016 en primer lugar; en segundo lugar tanto en el 2015 como en el 2016 16\% de ambas series procedieron de San Juan de Lurigancho y $8 \%$ en tercer lugar procedieron de Carabayllo en el 2015, mientras que en el 2016 el tercer lugar de procedencia fue el distrito de San Miguel.

En el Gráfico 4 apreciamos el tipo de nacimiento, predominando el nacimiento por cesárea 141 casos (60 \%) en el 2015 y 176 casos (61 \%) en el 2016.

De acuerdo a la distribución por género, se apreció que el mayor número de defunciones fueron de sexo masculino con 132 casos (56\%) para el 2015 y en el 2016 no se apreció diferencia entre los 2 géneros. Gráfico 5 .

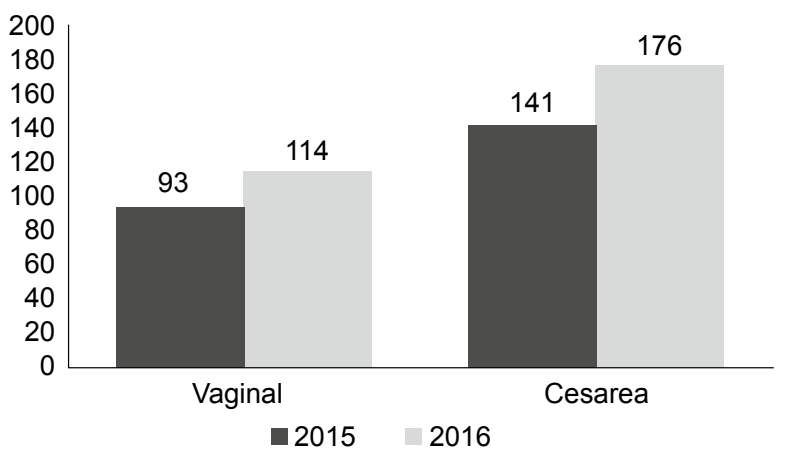

Gráfico 4. Tipo de nacimiento de fallecidos INMP 2015 $-2016$

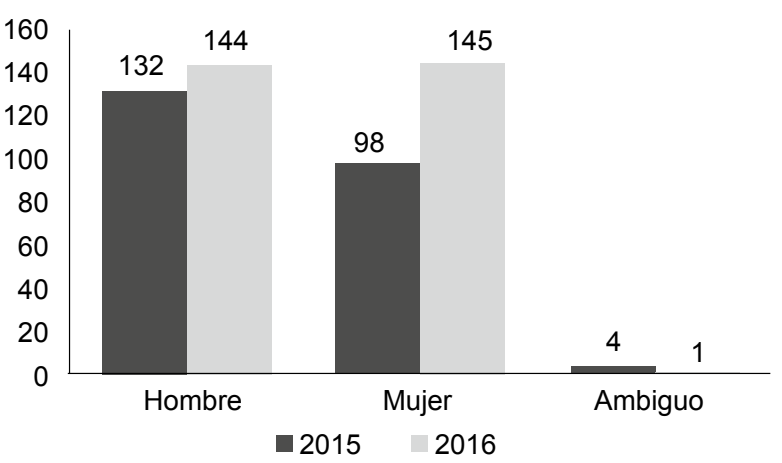

Gráfico 5. Estratificación por género - fallecidos INMP $2015-2016$

La distribución de acuerdo a la edad gestacional fue en ambos años a predominio de los menores de 37 semanas de edad gestacional (EG), para el 2015: 166 casos $(71 \%)$ y para el 2016: 211 casos (73\%). Destacando dentro de los prematuros, los menores de 28 semanas (35-37\% para el 2015 y 2016 respectivamente) y los prematuros tardíos (34 a 36 sem) 34\% en ambos años. Gráfico 6.

\section{5}
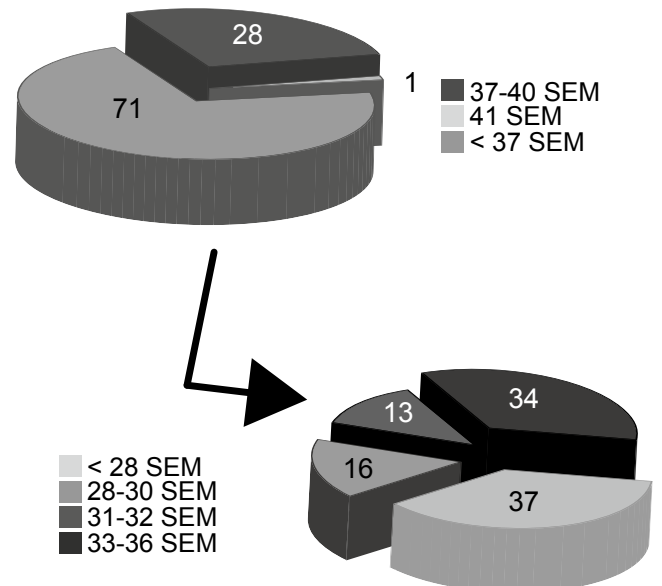

Gráfico 6ª . Edad Gestacional de fallecidos INMP 2015

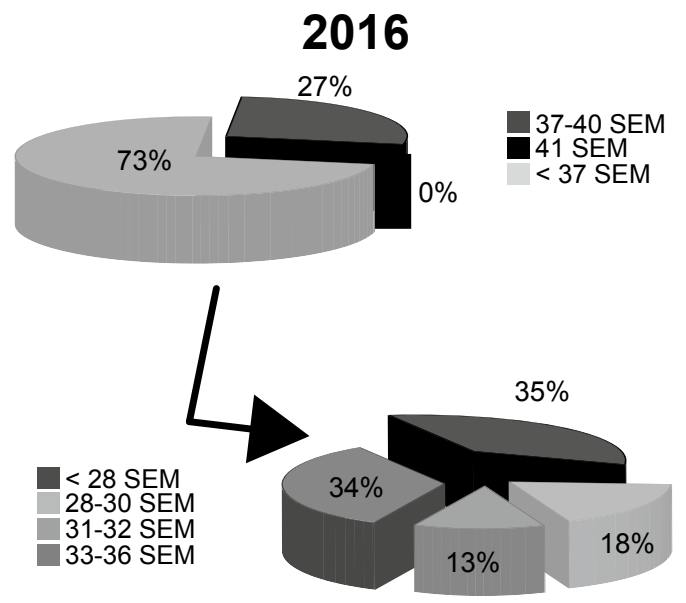

Gráfico 6b. Edad gestacional de fallecidos INMP 2016 
En el Gráfico 7 apreciamos la distribución por edad al momento de fallecimiento, para el 2015, 16\% (38/234) de los pacientes fallecieron dentro de la primera hora de vida con respecto al 15\% (43/290) en el 2016. Dentro de las primeras 24 horas de vida $46 \%(107 / 234)$ en el 2015 y $41 \%(119 / 290)$ en el 2016 . El $68.4 \%(160 / 234)$ fallecieron dentro de los primeros 7 días en el 2015, mientras que en el 2016 el $67.9 \%$ (197/290) falleció en este período.

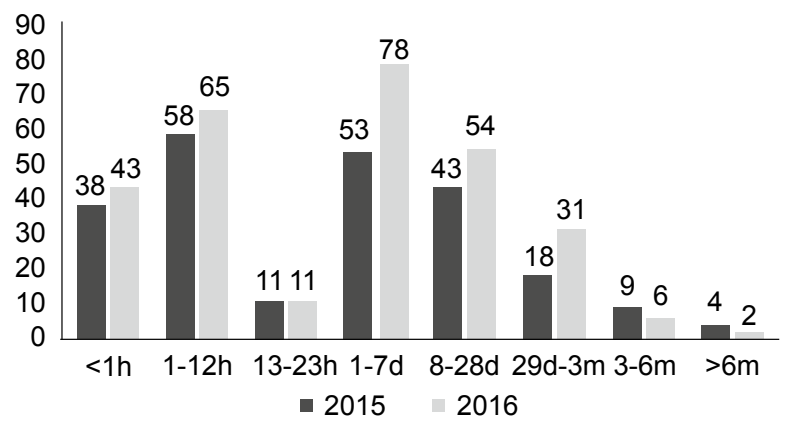

Grafico 7. Edad al momento de fallecimiento-INMP 2015 $-2016$

Con respecto a los pesos al nacer en ambas series en estos años, apreciamos que los menores de $1500 \mathrm{gr}$ eran en el 2015: 45\% (106/234) y 50.7\% (147/290) en el 2016. De este grupo en el $2015,50 \%$ eran menores de $750 \mathrm{gr}$ y en el 2016: 42\%. Gráfico 8.

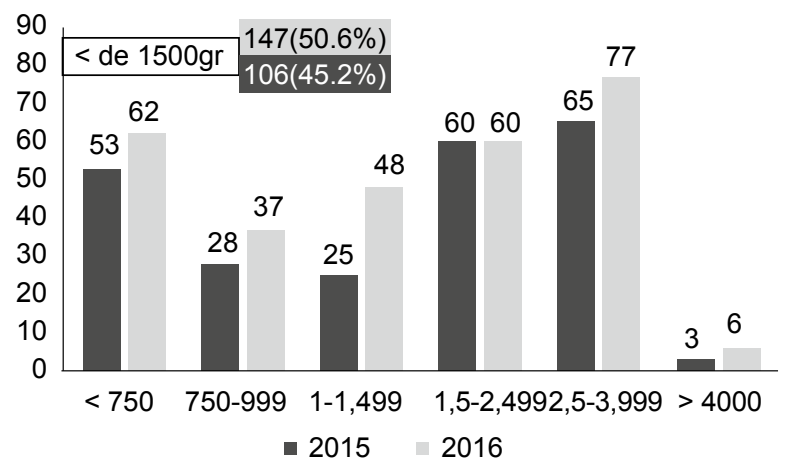

Grafico 8. Distribución de fallecidos por peso-INMP 2015 $-2016$

Con respecto a la causa básica de muerte: En el 2015 la principal causa de muerte fue: malformaciones congènitas $47,4 \%(111 / 234)$, quedando luego las infecciones $25,2 \%$ (59/234), asfixia y/o evento hipóxico severo 17,5\% (41/234) y prematuridad 8,5\% (20/234). En el 2016 la primera causa básica de fallecimiento fue malformaciones congénitas $48,6 \%$ (141/290), luego infecciones $23,1 \%$ $(67 / 290 \%)$, asfixia y/o evento hipóxico severo 10,7\% $(31 / 290)$ y prematuridad $11,4 \%$ (33/290). Gráfico 9. Las malformaciones congénitas más frecuentes fueron las del sistema nervioso central (holoprosencefalia, anencefalia, acranea, hidrocefalia congénita, mielomengocele, encefalocele, etc), seguidas por las malformaciones del aparato digestivo y cardiovascular. Tabla 2.

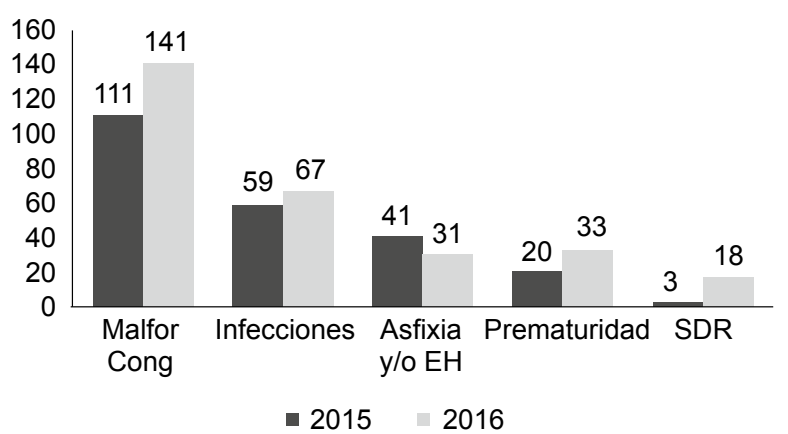

Grafico 9. Causas básicas de muerte-INMP 2015 - 2016

Tabla 2. Malformación congénita en fallecidos - INMP 2015-2016

\begin{tabular}{lcc}
\hline \multicolumn{1}{c}{ Malformaciones congénitas } & $\mathbf{2 0 1 5}$ & $\mathbf{2 0 1 6}$ \\
\hline Sistema nervioso central & 27 & 50 \\
\hline Aparato digestivo & 40 & 33 \\
Aparato respiratorio & 15 & 10 \\
\hline Renal & 18 & 10 \\
Musculo esquéletico & 11 & 9 \\
\hline Cromosomopatías & 9 & 39 \\
Cardiovascular & 31 & 49 \\
\hline Malformaciones congénitas múltiples & 13 & 26 \\
Total & $\mathbf{1 6 4}$ & $\mathbf{2 2 6}$ \\
\hline
\end{tabular}

Las morbilidades asociadas fueron múltiples: shock séptico, sepsis, trastorno de coagulación, enterocolitis necrosante, neumonía, insuficiencia renal, encefalopatía, hemorragia intraventricular ó intracerebral, anemia, hipertensión pulmonar, entre otras y se muestran en la Tabla 3.

Tabla 3. Principales causas de morbilidad asociada INMP 2015- 2016

\begin{tabular}{lrr}
\hline \multicolumn{1}{c}{ MORBILIDAD ASOCIADA } & $\mathbf{2 0 1 5}$ & $\mathbf{2 0 1 6}$ \\
\hline Sepsis & 87 & 144 \\
Neumonía & 20 & 18 \\
Trastorno de coag. & 18 & 56 \\
Encefalopatía & 15 & 17 \\
Hemorragia intraventricular & 14 & 19 \\
Enfermedad membrana hialina & 13 & 18 \\
Hipertensión pulmonar & 10 & 15 \\
Insuficiencia renal & 9 & 1 \\
Hemorragia pulmonar & 7 & 8 \\
Enterocolitis necrosante & 66 & 13 \\
Anemia & 2 & 40 \\
Neumotórax & - & 13 \\
Asfixia & - & 16 \\
Shock séptico & - & 38 \\
Insuficiencia respiratoria & - & 23 \\
Disglicemia & - & 23 \\
\hline
\end{tabular}

Entre las diferentes causas de defunciones neonatales, algunas será muy difíciles de intervenir a corto plazo, mientras que otras podrían ser reducibles (Gráfico 10), como las infecciones y asfixia, en función del conocimiento científico y la tecnología disponibles en nuestro medio. 


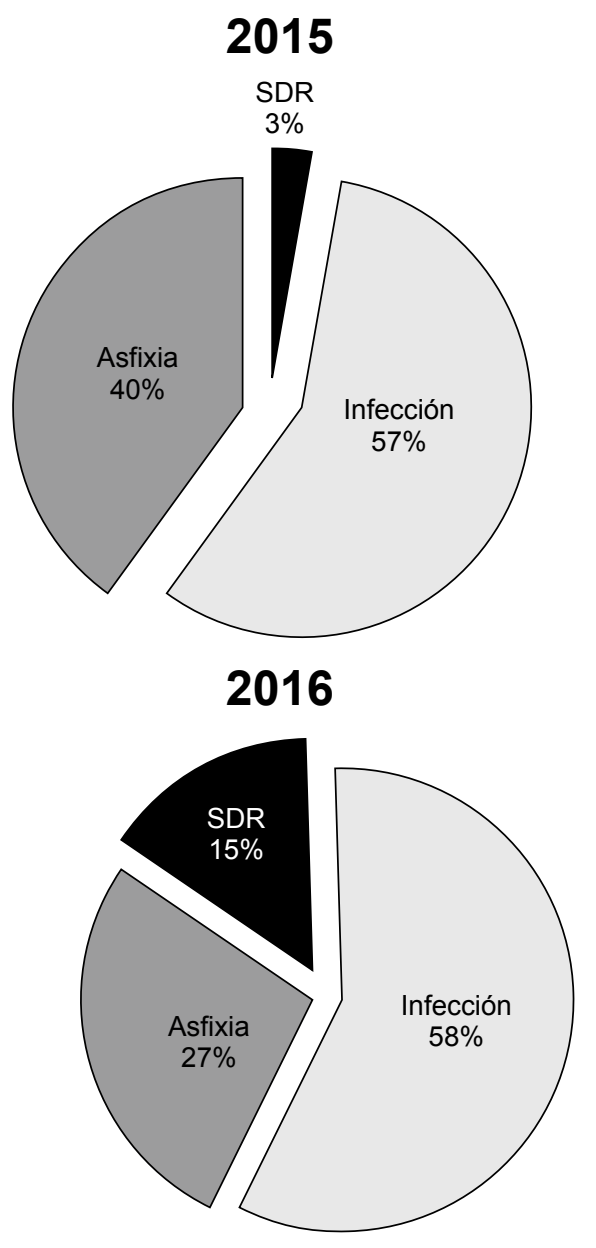

Grafico 10. Causas básicas de mortalidad reducibles IMNP-2015 2016

\section{DISCUSIÓN}

El INMP atiende el mayor número de partos de Lima y del Perú, en los últimos 10 años la mortalidad neonatal mostraba una disminución constante, sin embargo esa tendencia se modificó en el 2016, año que muestra un incremento de tasa de mortalidad neonatal en relación al 2015 en 2.5 x $1000 \mathrm{rnv}(22.6 \%)$.

Las cifras de mortalidad mundo que reporta el Banco Mundial, en 1990 actualizados al 2015 la mostramos en la Tabla 4, en la que se muestra un descenso en todos los países reportados, aunque en esta estadística no aparece Perú, nuestro resultado se ubicaría en el nivel reportado para América Latina: 8,56-11,06 x 1000 rnv. ${ }^{5}$

Centros latinoamericanos, como 05 Maternidades del sector público argentino, reportan para 2012, de 22, 324 rnv y más 651 recién nacidos derivados, 238 muertes neonatales, TM global 10.35 x 1000 rnv. $^{6}$ El incremento observado en nuestra tasa de mortalidad neonatal, podría ser explicado por incremento en el número de casos
Tabla 4. Tasas de mortalidad en países del mundo 1990 $-2015$

\begin{tabular}{lccllcc}
\hline \multicolumn{1}{c}{ PAIS } & $\mathbf{1 9 9 0}$ & $\mathbf{2 0 1 5}$ & \multicolumn{1}{c}{ PAIS } & $\mathbf{1 9 9 0}$ & $\mathbf{2 0 1 5}$ \\
\hline Canada & 4 & 3 & USA & 6 & 4 \\
Alemania & 3 & 2 & Francia & 4 & 2 \\
Costa Rica & 9 & 6 & Singapur & 4 & 1 \\
Angola & 59 & 49 & Japón & 3 & 1 \\
Bolivia & 42 & 20 & México & 21 & 7 \\
Brasil & 24 & 9 & Nicaragua & 24 & 10 \\
Camboya & 41 & 15 & Panamá & 17 & 10 \\
Chile & 9 & 5 & Venezuela & 13 & 9 \\
China & 30 & 6 & Africa Sur Sahara & 46 & 29 \\
Colombia & 18 & 9 & América Latina-Caribe & $\mathbf{2 2}$ & $\mathbf{9}$ \\
Cuba & 7 & 2 & Oriente Medio-Norte & 28 & 13 \\
Argentina & 15 & 6 & España & 7 & 3 \\
Ecuador & 24 & 11 & & & \\
\hline
\end{tabular}

Fuente: Banco Mundial 2015.

de malformaciones congénitas, según se aprecia en la tabla $\mathrm{N}^{\circ} 2$ donde para el 2015 se evidencia 111 malformaciones $(47.4 \%)$ en relación a 141 casos en el $2016(48.6 \%)$. Es así que la importancia relativa de las malformaciones congénitas ha aumentado (30 casos más) en este período. Esto se debe, en parte a, que las mejores posibilidades de diagnóstico e intervención prenatal permiten el nacimiento de fetos que antes morían intraútero, llegando a corrección quirúrgica, de sus anomalías. Sin embargo es indudable que el diagnóstico prenatal de algunas malformaciones susceptibles de corrección quirúrgica, es uno de los factores que más puede contribuir a reducir la mortalidad causada por esta afecciones, pero haciendo que estos niños nazcan en centros idóneos y dotados con los medios necesarios para corregirlos; problema aún por resolver en nuestra realidad, en especial en casos de cardiopatías complejas y pacientes que requieren neurocirugía.

En la literatura revisada en la Universidad Católica de Chile: $65.27 \%$ (47/72) de sus fallecidos correspondieron a malformaciones congénitas. ${ }^{7}$ Fariña y cols, en el análisis de mortalidad en 05 unidades neonatales de Argentina evidenció las malformaciones como la primera causa de mortalidad ( $3 / 5$ unidades), siendo la segunda causa en este estudio las infecciones ( $3 / 5$ unidades). ${ }^{8}$

El otro gran grupo de causas de mortalidad son las infecciones: 59 casos de sepsis en el 2015 a 67 casos en el 2016, este año con 38 casos de shock séptico y con incremento de casos de CID de 18 en el 2015, a 56 en el $2016 .{ }^{9}$ Asfixia y lo evento hipóxico severo se constituyó en la tercera causa de mortalidad con 41 casos el 2015 a 31 en el 2016, San Prematuridad representó causa de fallecimiento en 20 casos en el 2015 y 33 en el 2016, es la cuarta causa básica de mortalidad, coincidiendo con lo que reportan otros estudios en los que a menor edad gestacional y menor peso al nacimiento se incrementa la mortalidad. ${ }^{10}$ 
Dentro de las medidas para disminuir la mortalidad neonatal, podemos intervenir en la salud materna, neonatal y desarrollo de redes de contención neonatal. La intervención en la salud materna debe partir desde el inicio de la edad reproductiva de la mujer, con enfoque intersectorial y corresponden a resultados a mediano y largo plazo. En cuanto a la intervención neonatal a corto plazo corresponde el control y prevención de infecciones; asì como la reducción de problemas asociados a asfixia. Causas en las que nos toca intervenir exhaustivamente en el presente 2017. ${ }^{11}$

\section{REFERENCIAS BIBLIOGRÁFICAS}

1. Ministerio de Salud. Plan Nacional para la reducción de la morbilidad y mortalidad neonatal en el Perú 2016 - 2021, en el contexto del Plan Mundial.

2. Avila J, Tavera M, Carrasco M. Mortalidad Neonatal en el Perú y sus departamentos, 2011 - 2012/ Ministerio de Salud LIma, Dirección General de Epidemiologia, 2013. 188 p.

3. OPS/OMS Perú. Mortalidad neonatal en Perú. Disponible en: ww.paho.org/per/index.php? option...mortalidad-neonatal-enPeru.
4. Fernandez S, Gutierrez G, Viguri R. Principales causas de Mortalidad infantil en Mexico : tendencias recientes.Bol Med Hosp Infant Mex 2012; 69(2):144-148

5. Vel \& Trends in Child Mortality. Report 2011. Estimates Developed by the UN Inter-agency Group for Child Mortality Estimation (UNICEF, WHO, World Bank, UN DESA, UNPD). Datos.bancomundial.org/indicador/S.

6. Fariña $D$, Rodriguez $S$, Valhinger $M$ et al. Mortalidad Neonatal: Análisis causa raíz .Revista del Hospital Materno Infantil Ramón Sardá Buenos Aires 2012; 31(3):117-124. Argentina.

7. Aguila A, Nazer J, Bentjerdt R. Mortalidad Neonatal en un Hospital Universitario de Chile. Rev Chil Pediatr 1998; 69(1):16-20.

8. Valdéz M, Camaño A. Causas principales de mortalidad neonatal en el Hospital San Juan de Dios (III nivel) San José Costa Rica. Congreso Virtual hispanoamericano de Anatomía patológica. 2005.

9. Carvalho L, Wanick S, De Albuquerque M. Causas básicas de las muertes neonatales en Brasil: Conocer para prevenir. Rev Cubana Pediatría 2006;78(4).

10. Meritano J, Tsavoussian L, Címbaro R. Evaluación de la mortalidad neonatal en la Ciudad Autónoma de Buenos Aires según la residencia de la población y la pertenencia al subsistema de salud. Disponible en: http://dx.doi.org/10.5546/ aap. 2016.405

ORCID iDs

Carmen Dávila Aliaga

https://orcid.org/0000-0001-9411-5703 Elina Mendoza lbañez

https://orcid.org/0000-0001-6285-4464 